\title{
ARCHITECTONIQUE RICHIRIENNE, PSYCHIATRIE PHÉNOMÉNOLOGIQUE ET ETHNO-PSYCHIATRIE
}

\author{
JOËLLE MESNIL
}

\begin{abstract}
The anti-relativist architectonic of Marc Richir allows us to rethink the foundations of psychiatry, more particularly the phenomenological psychiatry of A. Tatossian and the non-relativistic ethno-psychiatry of G. Devereux in their respective articulation with psychoanalysis. Instead of seeing the unconscious of psychoanalysis as universal, Richir sees it as the symbolic unconscious of our culture based first of all on the symbolic institution of philosophy. Marc Richir is thus situated in an anti-relativist perspective, which nevertheless takes into account the relativity of cultures. While a relativist says that psychic pathology is defined by reference to the sociocultural norm in every society, Marc Richir would say that it is defined in relation to a non-realization of certain psychic processes on a transcendental level that is a common fund to all humanity, a phenomenological basis which by architectonic transposition will give rise to various symbolic institutions. There is no human without a phenomenological anchorage, but no human either without a symbolic institution and every symbolic institution, in the sense of culture and even of civilization, generates a symbolic unconscious of its own.
\end{abstract}

\section{Introduction}

La phénoménologie de Marc Richir se distingue de toute autre par une attention particulière portée aux registres architectoniques. Or, s'il est un domaine dans lequel les questions de méthode et d'architectonique se posent avec une acuité particulière, c'est bien la psychopathologie qui constitue justement un domaine privilégié chez le philosophe. Nous avons déjà interrogé ailleurs ${ }^{15}$ sa relation à la

15 Mesnil Joëlle, L'être sauvage et le signifiant. Marc Richir et la psychanalyse, MJW Fédition, 2018. ; "Penser la psychopathologie avec Marc Richir », in : Arrien, Sophie-Jan, Hardy, Jean-Sébastien Creative Commons Attribution License (http://creativecommons.org/licenses/by/4.0). 
psychanalyse. Nous aborderons donc ici plus spécifiquement l'ethnopsychiatrie et la phénoménologie psychiatrique avec G. Devereux at A. Tatossian. Il s'agira donc de mettre en évidence la pertinence et l'utilité de l'architectonique de Marc Richir lorsqu'on la mobilise dans un champ spécifique que lui-même n'a pas abordé : l'ethnopsychiatrie. Nous interrogerons donc longuement les pensées respectives de G. Devereux et A. Tatossian, sur la base de citations suffisamment consistantes pour pouvoir faire travailler la pensée de M. Richir dans l'approche critique de leurs conceptions. Nous serons ainsi amenés à utiliser les outils conceptuels que nous offre le philosophe pour aborder d'autres pensées que la sienne. D'une part, il s'agira de mettre en évidence en quoi, plus particulièrement, son architectonique permet de sortir de certaines impasses théoriques dues à des insuffisances dans la différenciation des niveaux ou des registres architectoniques mis en jeu non seulement dans l'étude des psychopathologies mais dans leur constitution même, d'autre part de faire apparaître que des psychiatres qui ne disposaient pas des différences conceptuelles qu'il a forgées ont pu néanmoins les mettre en œuvre de façon non thématisée. Ce qui importe ici n'est donc pas, répétons-le, la spécificité de la psychopathologie telle que Marc Richir l'a pensée mais celle de son architectonique dont nous pensons qu' elle gagnerait à être mobilisée dans n'importe quelle approche relevant d'une science humaine.

Interroger l'architectonique de Marc Richir pour aborder des questions que pose l'ethnopsychiatrie nous a paru d'autant plus approprié que sa perspective est résolument anti-relativiste alors même qu'il accorde la plus grande importance à la relativité des cultures. En ce sens, Richir nous met en garde contre une confusion entre la relativité culturelle et le relativisme culturel notamment au cours de l'établissement d'un diagnostic. Ces questions étant trop souvent posées de façon générale, voire superficielle, qui plus est dans le contexte d'affrontements idéologiques plus que de pensées élaborées, il importait d'offrir au lecteur une matière concrète qui invite à la réflexion sur la base de connaissances et d'exemples euxmêmes concrets où deux psychiatres posent la difficile question du diagnostic d'un patient d'une autre aire culturelle que la leur. Si nous avons retenu les textes de Devereux et de Tatossian, c'est qu'eux-mêmes ont une sensibilité aigue aux registres architectoniques même s'ils ne les thématisent pas. C'est alors lorsqu'on accorde

et Perrier, Jean-François (Ed.), Aux marges de la phénoménologie. Lectures de Marc Richir, Paris, Herman, 2019; " La notion d'existential symbolique chez Marc Richir: Vers un nouveau réalisme phénoménologique en psychopathologie ", in Annales de Phénoménologie - Nouvelle série, XVI, 2017 ; « Réflexion sur la notion de monade psychique. Une difficulté de lecture d'un texte de Marc Richir : «Phantasia, imagination, affectivité », in Annales de Phénoménologie, XV, 2016 ; « La pulsion chez Marc Richir », in Eikasia 47, 2013, pp. 527-572 ; «L'anthropologie phénoménologique de Marc Richir », in Revue Internationale de psychopathologie, XVI, 1994, pp. 643-664. 
la plus grande attention aux distinctions qu'ils mettent en œuvre dans l'écoute du discours de leur patient que l'on s'aperçoit que certaines distinctions architectoniques richiriennes, mais pas toutes, sont déjà en fonction chez eux.

Il est de fait que de l'établissement d'un diagnostic lui-même tributaire d'une certaine conception de ce qu'est la maladie, à celui de la mise en place d'une thérapie, une suite de difficultés se présentent, de risques de malentendus qui tiennent le plus souvent à des erreurs architectoniques. Il convient en effet chaque fois, avec chaque nouveau patient, de savoir se situer dans les registres architectoniques engagés dans la constitution de la maladie, puis tout au long de la thérapie envisagée, de continuer à s'y repérer avec une certaine assurance. Mais comment fait-on cela? En recourant à quelle méthode?

Notons qu'une telle méthode dépend étroitement de son «objet» et on ne saurait en cette matière la concevoir comme l'application d'un corpus théorique à une situation particulière. En effet, toute psychopathologie et toute psychothérapie, dès lors qu'elles mobilisent la phénoménologie, mais on va voir que c'est d'une autre façon le cas en ethnopsychiatrie, reposent incontournablement sur l'épochè de savoirs constitués en corps de connaissances. La question qui se pose alors encore et toujours est de trouver une façon de faire qui implique une attitude plus qu' elle n'engage des savoirs au moment même où pourtant elle ne peut jamais s'abstraire tout à fait de ces derniers. Le rapport de la phénoménologie à la psychopathologie n'est pas d'application, il ne peut principiellement pas l'être, mais d'implication. Il nous a semblé que si depuis le début des années vingt au siècle dernier, de nombreux travaux de psychopathologie ont accordé une importance particulière au courant phénoménologique, il leur a souvent manqué une rigueur architectonique ${ }^{16}$.

S'il n'y a pas une mais des phénoménologies, celle de Marc Richir a toujours exclu, on l'a dit, le relativisme que ce soit implicitement comme une conséquence en quelque sorte logique de son élaboration de pensée, ou même à maintes reprises explicitement, par exemple lorsque dans l'avant-propos à Phénoménologie en esquisses, il fustige le " "post-moderne» " où sous couvert d" "herméneutique", de "relativisme" ou de "perspectivisme" en lequel toutes les interprétations se vaudraient, à peu près tout paraît pouvoir être légitimement dit à propos de n'importe quoi ${ }^{17}$. " Il s'agit

16 Proposons à titre d'exemple, la confusion architectonique fréquente sur laquelle repose l'idée qu'il y aurait une homologie entre l'épochè phénoménologique et la "perte de l'évidence naturelle» chez le schizophrène. Contrairement à ce qui est dit fréquemment, la différence entre les deux ne tient pas tant au fait que l'épochè philosophique est volontaire et la perte de l'évidence naturelle involontaire, mais au fait que l'épochè phénoménologique reconduit au préréflexif qui est précisément ce que le schizophrène a perdu. Il y a donc là une vraie erreur architectonique. On confond alors Lebenswelt et monde de l'attitude naturelle.

17 Richir Marc, Phénoménologie en esquisses, Nouvelles fondations, Grenoble, Millon, 2000, p. 5. 
pour le philosophe de compter avec une ou des instances critiques à même d'offrir une limite à ce qui peut être dit dans une interprétation. Il y a des interprétations vraies et d'autres fausses, - notons que Marc Richir préfère dire qui « tombent juste» ou pas. Mais alors vraies ou fausses, tombant juste ou pas, par rapport à quel critère?

Il est vrai que chaque thérapeute se trouve confronté à cette intrication de registres architectoniques, et de façon renouvelée avec chaque patient singulier, c'est à première vue de façon encore plus évidente et problématique quand le patient vient d'une culture différente de la sienne. Il s'agit pourtant d'une «difficulté facilitante» (comme l'est le transfert en psychanalyse) puisque comme Devereux le note, la neutralité culturelle du thérapeute est plus facile avec un patient d'une autre culture. On retrouve ici une idée non équivalente mais homologue à celle de Richir selon laquelle lorsque nous abordons l'étude de la pensée mythique - une autre forme d' «exotisme» pour un regard philosophe -, nous pratiquons spontanément l'épochè phénoménologique hyperbolique.

Nous ne partirons pas ici de définitions, c'est à dire de ce que le philosophe entend par architectonique, et dans la foulée par réduction architectonique et d'épochè phénoménologique hyperbolique, les deux piliers de sa méthode phénoménologique. Nous amènerons ces notions dans le cours de la réflexion quand la nécessité s'en fera sentir. Il apparaîtra d'autant mieux que la phénoménologie n'est pas une doctrine mais un ensemble de questions. Nous verrons que même chez A. Tatossian - mais aussi chez G. Devereux auquel il se réfère à maintes reprises -, subsistent certaines ambiguïtés que l'architectonique richirienne nous a paru à même de dissiper. Il est d'ailleurs remarquable qu'on puisse déceler chez l'ethnopsychiatre une dimension phénoménologique, alors même qu'il ne s'est jamais référé à la phénoménologie. Nous verrons aussi que la conscience même des difficultés que pose la notion d'application méthodique de la phénoménologie à une situation clinique, déjà souvent exprimée ici et là, a presque aussi souvent buté sur de nouveaux obstacles sans parvenir à les dépasser, obstacles qui ont eux aussi une origine architectonique. Cet article pose ainsi les bases d'une étude encore en cours de l'apport de Marc Richir à la psychiatrie et à l'ethnopsychiatrie. Il s'inscrit dans le contexte d'un travail de plus grande ampleur où il s' agit de relire de grands textes de psychopathologie du point de vue anti-relativiste du philosophe en étant informé de l'architectonique qui soutient toute sa pensée ${ }^{18}$.

$18 C f$. "Constructions spéculatives et "constructions" phénoménologiques dans l'espace de la psychothérapie : Pour une critique de la notion de "construction" en analyse à partir de l'exemple de Serge Viderman ", in Annales de Phénoménologie, XIV, 2015 [cet article propose une lecture richirienne de textes du psychanalyste S.Viderman, fortement marqué par un hyper-relativisme souvent dénoncé par ses pairs mais sans arguments suffisants]. 


\section{Culturalisme versus anti-relativisme culturel en ethnopsychiatrie. Le point de vue d'A. Tatossian}

Que faut-il entendre exactement par relativisme culturel? À première vue, la réponse est simple et pourrait tenir dans l'aphorisme pascalien : "Vérité en deçà des Pyrénées, erreur au-delà». La notion de vérité serait donc relative à une aire culturelle. Le problème est que dès que l'on aborde des questions concrètes, notamment en ethnopsychiatrie les choses se compliquent singulièrement. Examinons la proposition suivante :

On nous rappelle à nous psychiatres étroitement occidentaux, que les états de transe n'ont rien d'illégitime sous d'autres cieux, que les hallucinations ne sont pas pathologiques chez les Indiens des Plaines, que la dissipation effrénée de ses biens dans un dessein de prestige social est une institution normale dans telle tribu indienne alors que nous parlerions de mégalomanie ou encore que chez certains Mélanésiens la suspicion régulière du voisin en cas de mauvaise récolte et du conjoint quand un individu meurt se rencontre et que nous aurions tort de diagnostiquer un délire de persécution. On en conclut que le même comportement [je souligne] est normal ou anormal au hasard des cultures, soit au relativisme culturel de ces notions. ${ }^{19}$

Le ton est ironique et il est clair que Tatossian ne partage pas les convictions des culturalistes comme Malinowski. Ce mélanésien, pour le psychiatre marseillais, peut délirer ou pas dans la mesure où le critère de pathologie n'est pas socioculturel, mais intrinsèque. Mais comment définir le registre «architectonique » dans lequel la notion même d' "intrinsèque» pourra avoir un sens, registre auquel on va se situer dès qu'on abandonne le critère socioculturel? Tatossian interroge alors la notion de comportement : «On ne saurait parler d'un "même comportement" chez le Mélanésien méfiant et le délirant occidental si l'on accepte que le vécu comme signification d'un comportement est partie intégrante et même déterminante de celui-ci ${ }^{20}$. À la différence de ce qu'on appelle comportement dans une perspective où seul le positif est pris en compte, par exemple, celle du DSM, plus que jamais dans sa cinquième et dernière version exclusivement attentive aux manifestations directement observables, le comportement ici n'est pas ce qui peut être observé et décrit objectivement parce qu'il inclut le vécu qui n’est rien de directement observable. Il se situe à un niveau architectonique, transcendantal

19 Tatossian Arthur, Psychiatrie phénoménologique, Paris, Acanthe, 1997, p. 131.

20 Ibid, p. 131-132. 
supposant justement une épochè des positivités. Pour Tatossian, "L'hypothèque du relativisme culturel n'atteint et ne met en danger que la psychiatrie fondée sur le symptôme, celle qui croit pouvoir réduire les tableaux cliniques à une somme de faits extérieurement observables et le diagnostic à leur relevé ${ }^{21}$. Alors que : «Pour le psychiatre, un comportement n'est pas anormal de par sa fréquence ou son degré d'adaptation sociale, mais de par sa signification et plus précisément de par sa signification individuelle ${ }^{22}$. En effet, précise Tatossian :

Toute la question est de savoir ce que veut dire «le même comportement». Si cela veut dire strictement les comportements matériels, directement observables, y compris la signification objective [je souligne] des gestes faits et des mots dits : ce sont effectivement les mêmes comportements chez le Mélanésien méfiant et le délirant occidental persécuté. Il est bien vrai que le même comportement est pathologique ici, normal là. Si le même comportement inclut le vécu, c'est-à-dire la signification subjective des gestes et des mots, c'est-à-dire si le comportement n'est pas seulement le symptôme d'un vécu considéré comme inaccessible mais inclut le vécu, c'est-à-dire la signification [je souligne], autrement dit, si le comportement est considéré comme phénomène global, ce n'est justement plus le même comportement. ${ }^{23}$

Ni la réalité factuelle ni la coutume ne peuvent ici servir d'instance critique. La position de Tatossian est clairement anti-relativiste. Il met en garde contre une confusion des niveaux d'approche : la façon dont telle culture interprète tel comportement ou telle manifestation en termes de normalité ou d'anormalité socioculturelle - c'est-à-dire de conformité ou de déviance -, ne se situe pas au même niveau que le fait proprement psychiatrique, non socioculturel. Tatossian s'oppose donc fermement à l'idée que «le même comportement est normal ou anormal au hasard des cultures, soit au relativisme culturel de ces notions ${ }^{24}$.

On reconnait bien la position de Georges Devereux absolument opposé à tout relativisme psychiatrique, contrairement à ce qu'on dit parfois, confondant sa position avec celle des culturalistes nord-américains qu'il combat. Pour l'ethnopsychiatre, il y a des «critères absolus de normalité » ${ }^{25}$. Mais comment les conçoit-il ?

\footnotetext{
Ibid, p. 132.

Ibid, p. 132.

Ibid, p. 208.

Ibid., p. 131.

Devereux Georges, Essais d'ethnopsychiatrie générale, Paris, Gallimard, 1970, p. 55. Devereux défend contre Malinowski la thèse de l'unité psychique de l'humanité. «D'un point de vue psychiatrique, les critères de normalité valables sont tous absolus, ie indépendants des normes d'une quelconque culture ou société, mais conforme aux critères de la Culture en tant que phénomène universellement humain.»
} 
C'est bien toute la question de l'instance critique qui est posée. Si l'on refuse le critère socioculturel, relatif, par rapport à quel critère «absolu» va-t-on définir la pathologie mentale? Qu'est-ce qui autorise à dire que tel patient est bel et bien psychiquement malade où qu'il vive et d'où qu'il vienne? Comment définir le délire ou l'hallucination? Comment comprendre ces mots de Griesinger ${ }^{26}$ que Tatossian reprend à plusieurs reprises à son compte : «Deux individus peuvent dire ou faire exactement la même chose, par exemple exprimer leur croyance à l'influence des sorciers, ou la crainte d'être damnés pour l'éternité; l'observateur qui sait ce que cela veut dire déclarera l'un de ces individus aliéné, et l'autre sain d'esprit ${ }^{27}$. Interrogeons-nous sur le sens qu' il convient de donner à l'expression : «l'observateur qui sait [je souligne] ce que cela veut dire». La phénoménologie ne nous intime-telle pas de mettre entre parenthèses tout préjugé ? En quoi consiste ce «savoir» de l'observateur? Une lecture attentive des travaux de Tatossian sur plusieurs dizaines d'années confirme que ce savoir se rapporte toujours d'une façon ou d'une autre au "vécu» du patient, à la «signification» de son comportement dont l'observateur va tenir compte dans son interprétation de la croyance «subjective» sous-jacente au comportement qu'il observe. Cette croyance subjective elle-même s'articule d'une certaine façon avec des croyances culturelles de la société dans laquelle il vit; il s'agit toujours de la façon dont il s'approprie subjectivement cette croyance instituée. Tatossian dit en effet que le comportement inclut le vécu, et que le vécu c'est la signification subjective des gestes et paroles que le psychiatre observe chez le patient ${ }^{28}$. Reste dès lors à savoir ce qu'il faut entendre par subjectif.

\section{La notion de vécu et celle de subjectivité}

Un exemple donné par Devereux (et connu de Tatossian) nous permettra de comprendre en quoi consistent le vécu et la signification subjective d'un propos, d'un geste, d'une expression : un indien raconte à l'ethnopsychiatre qu'il a quitté sa mère dans la vallée où elle est restée, puis qu'il a gravi la montagne en haut de laquelle sa mère l'attendait : l'ethnopsychiatre qui connait bien la culture de l'indien a alors l'idée de lui demander «quelle mère» il a quittée et "quelle mère» il a trouvée : était-ce bien la même personne? Il apparaît que ce n'est pas le cas. Alors, seule la connaissance de l'ethnologue a permis d'éviter de diagnostiquer un délire

26 Griesinger est un psychiatre allemand né en 1817 à Stuttgart, décédé en 1868 à Berlin.

27 Griesinger, cité par Tatossian A., Psychiatrie phénoménologique, op. cit., p. 132.

28 Voir entre autres l'équivalence entre vécu et signification du comportement in Tatossian A., Psychiatrie phénoménologique, op. cit., pp. 208-209. 
là où il n'en était pas question! Cette mère du haut de la montagne était une sœur de sa «vraie» mère, mais il l'appelait «mère» conformément à la terminologie de sa tribu. S'il tient compte du fait que la personne qu'il dit avoir quittée et celle qu'il trouve n'est pas la même, le psychiatre considère que cet homme ne délire pas. On ne peut donc pas, partant de l'anti-relativisme de Devereux, conclure que la culture du patient ne doit pas être prise en considération; au contraire, dans ce cas, c'est la connaissance du système de parenté du sujet qui lui a fait poser une question qui sinon ne lui serait pas venu à l'esprit. On notera toutefois que si Devereux a pris en considération la culture du patient, c'était pour la mettre entre parenthèses: comme Tatossian, il considère que «Pour le psychiatre, l'intérêt majeur de la connaissance particulière est de lui permettre de mieux les neutraliser [je souligne] et de supprimer un obstacle vers sa véritable tâche ${ }^{29}$. Cette neutralisation leur permet d'accéder à un registre architectonique sous-jacent à la culture. C'est-à-dire que contrairement au culturaliste relativiste, Devereux comme Tatossian vont tenir compte dans leur interprétation de la culture du patient non pas positivement mais négativement.

On notera toutefois que Devereux recourt ici subrepticement si l'on peut dire au critère de la réalité empirique où l'ubiquité est impossible. C'est-à-dire qu'il joue en quelque sorte la réalité empirique contre la culture; une épochè ayant été accomplie, une autre reste à faire sans qu'il n'en dise rien. On sait que Devereux s'appuyait dans sa pratique ethnopsychiatrique sur la psychanalyse; or, il est vrai que d'un point de vue freudien la réalité empirique ne constitue pas un critère déterminant pour trancher ou pas en faveur du délire. Ici, la culture du patient a à la fois été prise en considération ET mise entre parenthèses, mais ce n'est pas le cas de la réalité factuelle qui est devenue un nouveau critère discriminant. Alors en quoi sa conclusion reste-t-elle malgré tout valide? Il peut être utile de rappeler que cet indien vit dans une culture où les hallucinations sont considérées comme normales. Ce qui autorise un rapprochement avec ce que Devereux nous dit ailleurs du «primitif» vivant dans une culture qui ne fait pas la différence entre réel et imaginaire : il ne saurait être considéré comme schizophrène s'il avoue un péché d'adultère commis seulement en rêve du fait précisément que dans sa société, rêve et réalité sont consubstantiels. Devereux rappelle que du point de vue historique cette frontière est récente; les Grecs furent, dit-il, parmi les premiers à distinguer réel et imaginaire en tant que catégories de l'esprit, à quoi il ajoute qu'ils ne cessèrent de la nier pratiquement. On pourra s'étonner. Devereux n'est-il pas pris ici en flagrant délit de relativisme? Non, car là encore, il convient d'être attentif à une essentielle différence architectonique. Dans le cas de cet aveu d'adultère, il faut bien voir que ce qui est en jeu c'est

29 Ibid., p. 134. 
une croyance culturelle selon laquelle commettre l'adultère est un péché même en rêve. Mais le rêveur quand il est éveillé sait bien qu'il a commis le péché en rêve. Il ne confond pas subjectivement ce qu'il fait en rêve et ce qu'il fait en «réalité». Cela rejoint la position de Richir pour qui dans une culture mythique, imaginaire et réel tendent à se confondre, ce qui n'empêche pas que dans les situations triviales, les membres de cette culture font à coup sûr la différence entre mensonge et vérité car cette différence est humaine. Mais si nous comparons ce dernier exemple des hallucinations culturellement admises et celui de l'indien aux deux mères, nous constatons qu'ils engagent des registres différents. Il faut en fait distinguer trois «choses » : la réalité empirique, la « réalité» culturelle et la « réalité» transcendantale et il est nécessaire de faire l'épochè des deux premières pour atteindre la troisième.

On peut se livrer à des variations sur la base de ces différents exemples : reprenons donc la situation du mélanésien évoqué plus haut. Elle pourrait être en effet comparée à celle du paranoïaque dans notre culture, mais d'un autre point de vue que celui qu'envisage Tatossian : tout comme d'un point de vue psychanalytique, on dit avec «humour» que «même le paranoïaque a des ennemis», on pourra dire que même le mélanésien soupçonneux de son voisin en cas de mauvaise récolte peut effectivement avoir un méchant voisin qui a empoisonné son champ; d'un point de vue psychanalytique, il pourra malgré tout effectivement délirer quand bien même la malveillance serait réelle, de la même façon qu'il pourra délirer, d'un point ethno-psychiatrique non relativiste, même si dans sa culture un tel soupçon repose sur une croyance instituée. Tout dépend dans chaque cas de la façon dont il s'approprie subjectivement tant un réel empirique qu'un élément de culture.

Si le raisonnement de Devereux concernant l'indien et ses deux mères est malgré tout valide c'est donc parce que la question essentielle se situe en réalité à ce moment là et dans cette situation par rapport à la possibilité d'envisager la croyance en l'ubiquité. Il se pourrait que dans telle autre culture on croie à l'ubiquité, alors, il faudrait se demander si le patient s'approprie subjectivement cette croyance ou pas. On voit qu'il s'agit de savoir se repérer à chaque instant par rapport à plusieurs registres architectoniques : celui de la perception, celui de la croyance culturelle et celui de la croyance subjective, et de savoir se déplacer avec une certaine agilité des uns aux autres. Viser juste.

Bien évidemment, quelqu'un qui dira avoir quitté une personne dans la vallée et retrouver cette même personne en haut de la montagne qu'il vient de gravir alors qu' elle est bel et bien restée en bas, et qui en sera intimement persuadé, sera délirant ou souffrira d'hallucinations partout, qu'il soit occidental, indien ou n'importe quoi d'autre. 
Prenons maintenant l'exemple du shaman qui pour Devereux est quant à lui effectivement malade d'un point de vue psychiatrique tout en étant parfaitement adapté à la société dans laquelle il vit; l'ethnopsychiatre est là encore en désaccord avec «le relativisme culturel [qui] confond croyance traditionnelle et expérience subjective. ${ }^{30}$ Devereux donne encore tout au long des Essais bien d'autres exemples qui montrent que "pour muer un matériau culturel en symptôme, il faut transformer une croyance courante, traditionnelle, en expérience subjective. $»^{31}$ Là encore, c'est toute la différence entre croire au paradis et dire qu'on y est allé, croire au royaume des morts et dire qu' on en revient, ou encore croire en Dieu et affirmer qu'on l'a vu dans sa chambre la veille au soir. Mais on doit être attentif au fait que dans le concret de la vie et dans le concret de l'entretien psychothérapeutique, ce niveau de croyance culturelle est toujours intriqué avec celui de la croyance en la réalité empirique. Il est nécessaire de pratiquer l'épochè de chacun de ces deux niveaux, mais on voit bien qu' on ne peut jamais le faire au même moment.

L'invocation de la dimension subjective engagée dans la réalité culturelle aussi bien qu'empirique appelle toutefois une réflexion. En effet de quelle subjectivité s'agit-il ? L'approche de G. Devereux qui n'est pas un psychiatre phénoménologue suscite pourtant les mêmes interrogations que celle de Tatossian qui dans les « Us et abus de la phénoménologie en psychiatrie ${ }^{32}$, s'inquiétait des «malentendus suscités par l'approche phénoménologique en psychiatrie. ${ }^{33} \mathrm{Il}$ attribuait la plupart de ceux-ci à la «confusion de l'approche phénoménologique avec un essai de réhabiliter la perspective subjective en psychopathologie, que ce soit pour l'en louer ou l'en blâmer. " ${ }^{34}$ Ce qui intéresse le "véritable» psychiatre phénoménologue, c'est nous avertit le psychiatre phénoménologue non pas les vécus psychologiques, le contenu de l'éprouvé psychologique, ce qu'aurait pu laisser penser la notion de «signification» mais «le mode de présence de l'objet délirant à la conscience du malade. " ${ }^{35} \mathrm{Il}$ s'agit là du vécu phénoménologique et non psychologique et ce vécu phénoménologique se situe à un registre transcendantal qui doit être atteint par l'épochè phénoménologique hyperbolique telle que Richir l'a conçue. C'est ce même registre qui est atteint par la réduction architectonique. On peut ainsi en prenant les manifestations pathologiques à la racine les envisager dans plusieurs directions selon les registres qu' elles mettent en œuvre. Par exemple, dans

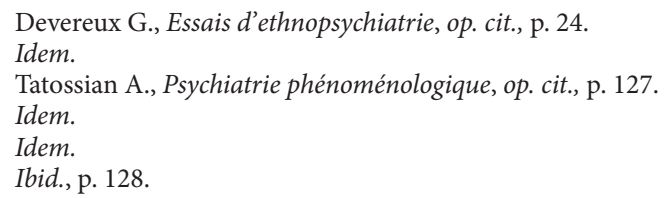


les Essais d'ethnopsychiatrie générale, Devereux consacre un chapitre à la schizophrénie comme désordre "ethnique ${ }^{36}$, et un autre à la schizophrénie comme désordre «type $»^{37}$. De quoi s'agit-il ?

\section{La notion de registre architectonique en psychiatrie et en phénoménologie richirienne}

Devereux distingue quatre sortes de "désordres » psychiques : les «désordres ethniques», les "désordres types», les désordres sacrés ou chamaniques et les désordres idiosyncrasiques. Il est impossible ici d'entrer dans toute la complexité de la pensée de Devereux mais donnons quelques précisions indispensables pour montrer qu'il est possible de détecter une homologie entre ces formes et les registres architectoniques tels que Marc Richir les conçoit.

Les «désordres ethniques " possèdent en général un nom, par exemple l'amok des Malais ou le bersek des Scandinaves. Ces troubles sont liés du point de vue de Devereux au fait que la culture fournit aux individus des "modèles d'inconduites" (Linton ${ }^{38}$ ). Les désordres ethniques et les conflits de base des désordres ethniques sont ainsi dépendants des modèles culturels. ${ }^{39}$ Les désordres ethniques peuvent également relever de la psychiatrie traditionnelle, mais c'est alors de par leur conformité au modèle culturel dominant dans telle ou telle culture. C'est ainsi que Devereux voit dans l'hystérie la névrose ethnique du temps de Charcot, et dans la schizophrénie «la psychose ethnique de notre temps. "40 Il observe également que le «masque culturel» peut servir à ne pas prendre conscience de la valeur subjective de problèmes œedipiens. ${ }^{41}$

La schizophrénie peut toutefois être considérée sous un autre angle qui engage plus qu'un simple point de vue puisqu'il n'est plus question alors de structuration

36 «La schizophrénie psychose ethnique» (1965) in Devereux G., Essais d'ethnopsychiatrie..., op. cit.

37 "Une théorie sociologique de la schizophrénie " (1939) in Devereux G., Essais d'ethnopsychiatrie, op. cit., p. 215.

38 Ralph Linton (1893-1953) est un anthropologue américain qui a créé le concept de "modèle d'inconduite» que Devereux a repris à son compte.

39 Ce que Devereux appelle «modèle culturel» apparaît au travers d'attitudes, d'émotions, de façon de vivre les choses; ils ne doivent pas être confondus avec ce qu'il nomme «traits culturels» qui renvoient aux techniques de soin, aux méthodes éducatives, à tout ce qui relève de l'apprentissage. En termes richiriens, ils ne se situent pas au même registre architectonique.

40 Notons toutefois que les Essais ont été publiés en 1970 et qu' ils comportent des textes rédigés bien avant.

41 Devereux donne l'exemple (Devereux G., Essais d'ethnopsychiatrie, op. cit., p. 47) d'un homme dont la mère blanche a épousé un Indien et qui dit qu'il hait son père parce qu'il est un Indien alors que pour le psychiatre-psychanalyste, dans la perspective de l'Edipe, c'est parce qu'il couche avec sa mère. 
seconde d'un contenu préalable, mais de processus sous-jacents effectifs. Ce qui nous amène à la deuxième sorte de désordre : la schizophrénie comme "désordre type» est une «vraie» schizophrénie engageant effectivement des processus psychiques qui caractérisent cette pathologie, alors qu'on l'a vu, la schizophrénie comme «désordre ethnique» peut n'en avoir que l'allure. Les «désordres types» sont selon Devereux fonction de la différence entre sociétés à solidarité mécanique et organique. ${ }^{42}$ Un membre d'une Gesellschaft deviendra dit-il plutôt schizophrène (intrinsèquement) et un membre d'une Gemeinschaft plus hystérique (intrinsèquement). Là, ce ne sont pas comme dans le désordre ethnique des modèles culturels qui orientent la pathologie mais la structure sociale envisagée principalement sous l'angle du rapport entre individu et société.

On voit donc qu' il importe dans chaque cas au psychiatre de savoir se situer au bon registre. A-t-il affaire à une «vraie» schizophrénie, c'est-à-dire à une pathologie qui met effectivement en œuvre un certain type de processus "psychiques» qui caractérisent ce qu'on appelle schizophrénie? Ou bien par exemple à une hystérie d'allure schizophrénique, c'est à dire à une hystérie se manifestant par des symptômes qui sont ceux que l'on considère habituellement comme étant ceux de la schizophrénie (dans ce cas, on s'attache non à la structure sous-jacente, au "vécu ", mais au symptôme) ? Ou encore, ne se laisse-t-il pas emporter par une tendance liée à la culture dans laquelle il vit à voir des schizophrènes là où il s'agit en réalité d'hystériques (ou d'autre chose)? On voit bien que dans chacun de ces trois cas de figure, on ne se situe pas au même registre architectonique.

Une troisième sorte de pathologie réside dans les "désordres sacrés». Le chamanisme en fait partie. Pour Devereux, le chaman est malade même s'il est adapté à sa culture et a un rôle social reconnu puisque dans cette culture il est aussi soignant : il fournit à ses patients des défenses culturellement appropriées qui permettent à ceux-ci de faire face à leurs conflits idiosyncrasiques. Il remplace les conflits et défenses idiosyncrasiques par des conflits culturellement conventionnels et par des symptômes ritualisés «sans qu'intervienne jamais de prise de conscience. $»^{43}$ En effet, pour Devereux, ethnopsychiatre psychanalyste, une vraie thérapie doit aboutir à une prise de conscience, et selon lui, seule la psychanalyse est à même de produire ce résultat. Nous reviendrons sur ce point.

Les «désordres idiosyncrasiques» sont quant à eux ceux que prend en compte la psychiatrie occidentale standard et que la psychanalyse a largement repris à son compte. Ils sont dans l'optique de Devereux la conséquence de traumatismes par

42 Cf. Devereux G., Essais d'ethnopsychiatrie, op. cit., pp. 64-65.

43 Ibid., p. 19. 
rapport auxquels la culture ne fournit pas de défenses ni de symptômes permettant de fixer l'angoisse.

On notera que chacune de ces quatre formes de «désordre» mettent en œuvre une articulation différente des registres architectoniques : symbolique et phénoménologique dans les termes de Richir. Par exemple, les désordres chamaniques sont enracinés dans l'inconscient ethnique (que dans la perspective de Devereux tous les membres d'une même culture ont en commun) à la différence des désordres ethniques qui sont enracinés dans le modèle culturel; ce dernier peut certes en tant qu'habitus être inconscient, mais en un tout autre sens du terme. Pour Devereux, ces formes couvrent la totalité de la psychopathologie humaine de tout temps et en tout lieu. De plus, «La psychanalyse parfois considérée et critiquée comme étude psychosociologique sur le terrain d'une certaine classe sociale d'indigènes viennois de la fin du 19 ème siècle, a en fait portée universelle. ${ }^{44}$ De son point de vue, seule la cure analytique est à même de guérir véritablement un patient de par la prise de conscience qu'elle réalise. ${ }^{45}$ Pourtant, il convient de s'interroger sur le registre architectonique auquel se situe cette universalité de la psychanalyse. $S$ 'agit-il de dire que l'inconscient «de la psychanalyse» se retrouve dans toute culture, ou qu' il se situe à un registre architectonique qui lui est universel mais qui peut prendre d'autres formes que «l'inconscient de la psychanalyse»?

On sait que Richir quant à lui a toujours vu dans la psychanalyse le «paradigme de l'anthropologie phénoménologique». Ce qu'il retient avant tout de la psychanalyse, c'est la méthode de l'attention flottante qui paraît bien correspondre à l'épochè phénoménologique hyperbolique puisqu' elle ouvre non plus à des aperceptions mais à des entre-aperceptions. Il en retient aussi l'attention portée aux processus primaires (dans ses termes, les «synthèses passives de premier degré») mis en œuvre dans le rêve mais aussi dans la pathologie. Ces processus caractérisent le mode de fonctionnement de l'inconscient. Mais en quel sens du terme? C'est ici que l'architectonique du philosophe l'amène à distinguer ce qui chez Devereux et Tatossian est encore confondu lorsqu' ils disent que «ce n'est qu'au niveau du sens et de l'inconscient freudien qu'est retrouvée l'unité psychique de l'humanité. ${ }^{46}$ La rigueur architectonique exige ici deux distinctions que ne font ni l'un ni l'autre : celle, anti-relativiste, entre inconscients phénoménologique et symbolique; et celle qui s'impose au sein de ce dernier puisque si l'on tient compte de la relativité des institutions symboliques, l'inconscient de la psychanalyse est un inconscient symbolique parmi d'autres possibles, celui de la culture dans laquelle

44 Tatossian A., Psychiatrie phénoménologique, op. cit., p. 133.

45 C'est une différence essentielle par rapport à son disciple et dissident Tobie Nathan.

46 Ibid., p. 133. 
nous vivons et qui selon Richir est tributaire de l'institution symbolique de la philosophie. Ce n'est pas par exemple l'inconscient d'une culture mythique : pour Richir, «L'inconscient de la psychanalyse n'est pas transhistorique et universel. » ${ }^{47}$

\section{Conclusion}

Alors que Tatossian et Devereux tout deux anti-relativistes prônent l'idée d'un universalisme de la psychanalyse et de l'inconscient qu'elle a découvert, l'anti-relativisme de Richir se double d'une conscience aiguë de la relativité du symbolique : «L'envers de notre démarche est bien une "relativisation" du symbolique, une ouverture à son historicité, qui est l'une des dimensions dominantes de notre temps. ${ }^{48}$ Mais il adopte une posture critique par rapport au fait que «celle-ci aboutit le plus souvent, aujourd'hui, à une sorte de "déperdition" symbolique, à un "relativisme" et à une indifférenciation générale " 49 . C'est précisément pour ne pas confondre relativité ou relativisation et relativisme qu'il est nécessaire d'être attentif au registre architectonique auquel on se situe dans chaque acte et dans chaque parole. L'anti-relativisme de Richir réside dans l'idée qu'il y a une base phénoménologique commune à toute l'humanité, l'inconscient phénoménologique ${ }^{50}$, qui est l'un des deux grands registres architectoniques qui traversent toute sa pensée. Tout n'est pas construit. Sur cette base, se met en forme un langage phénoménologique, puis par transposition architectonique, des institutions symboliques (par exemple, le mythe, la philosophie (dans laquelle prend place l'institution de la psychanalyse), les religions, la démocratie), chacune laissant derrière elle une forme d'inconscient symbolique qui lui est propre. L'unité psychique de l'humanité que Tatossian et Devereux situent au registre de l'inconscient de la psychanalyse se situe donc pour Richir dans l'inconscient phénoménologique et dans l'inconscient symbolique d'extension plus large que l'inconscient de la psychanalyse qui n'en est qu'une figure possible.

Nous avons là un exemple qui montre à quel point la distinction entre registres phénoménologique et symbolique est nécessaire à toute psychiatrie, notamment à l'ethnopsychiatrie et en quoi l'épochè phénoménologique hyperbolique telle

\footnotetext{
Richir Marc, «Affectivité sauvage, affectivité humaine : animalité et tyrannie», in Epokhé, VI, L'animal politique, Grenoble, Millon, juin 1996, pp. 75-115, p. 109.

48 Richir M., Phénoménologie et institution symbolique, Grenoble, Millon, p. 375.

49 Idem.

50 Il convient de ne pas confondre cet inconscient phénoménologique avec un inconscient collectif puisqu'à la différence de ce dernier, il se situe en deçà de tout thème fixé.
} 
que Richir l'a conçue peut dans chaque cas permettre de remonter en deçà de la culture pour reconduire à la dimension phénoménologique qui est sa base transcendantale. Il s'agit non seulement d'atteindre comme nous y invitait Devereux LA culture en mettant entre parenthèse, LES cultures, mais plus radicalement d'être conscient que l'épochè doit remonter en amont de la culture elle-même jusqu'à une dimension phénoménologique que le psychiatre n’a pas pensée en tant que telle, ce qui l'amène à attribuer de façon unilatérale à la culture, c'est-à-dire à l'institution symbolique, ce qui n'en relève que pour partie. LA culture est universelle en ce sens qu'il n'y a pas d'homme sans institution symbolique, mais il n'y a pas non plus d'institution symbolique sans ancrage phénoménologique. Cet ancrage est ce qui rend possible le recul typique de l'humain par rapport à son expérience, recul qui est précisément compromis par les pathologies psychiques. Certes, un ethnopsychiatre peut avoir l'intuition de ces différences architectoniques sans les thématiser, mais le problème est que dans le cadre de son enseignement, c'est le thème qui sera transmis et non l'intuition, en sorte qu'un étudiant moins «intuitif " pourra aisément se fourvoyer. Cela pourra avoir de fâcheuses conséquences car la base architectonique qu'il s'agit de toucher ne relève pas d'une spéculation mais d'une «réalité», qui pour être transcendantale n'en est pas moins contraignante comme le souligne Richir : dimension symbolique et dimension phénoménologique jouent effectivement le rôle d'instance critique l'une par rapport à l'autre. Comme le note Pablo Posada Varela : «L'architectonique [...] est bien plus qu’une démarche théorique, dans la mesure où elle trouve, dans l'expérience elle-même, un répondant, c'est-à-dire quelque chose qui est intrinsèquement architectonique. ${ }^{51} \mathrm{C}^{\prime}$ est cet intrinsèque qui sera l'ultime instance critique permettant de discerner ce qui dans n'importe quelle culture est pathologique de ce qui ne l'est pas.

Joëlle Mesnil, après des études de Philosophie, d'Anthropologie, de Langues scandinaves et de Psychologie, a soutenu à Paris 7, en 1988, une thèse pluridisciplinaire sur «La désymbolisation dans la culture contemporaine». Elle a exercé pendant une vingtaine d'années le métier de psychologue clinicienne dans un contexte hospitalier avec des patients psychotiques. Les recherches qu'elle poursuit sur la «désymbolisation» qu'elle préfère désormais nommer «dé-phénoménalisation» sont étroitement liées à son étude de la pensée de Marc Richir.

51 Posada Varela Pablo, «Épochè hyperbolique et réduction architectonique», in : Arrien, Sophie-Jan, Hardy, Jean-Sébastien et Perrier, Jean-François Perrier (Ed.), Aux marges de la phénoménologie. Lectures et percées dans l'œuvre de Marc Richir, Paris, Hermann, à paraître. 